\title{
Correction to: Cheese consumption and risk of cardiovascular disease: a meta-analysis of prospective studies
}

\author{
Guo-Chong Chen ${ }^{1} \cdot$ Yan Wang ${ }^{2} \cdot$ Xing Tong $^{1} \cdot$ Ignatius M. Y. Szeto ${ }^{2} \cdot$ Gerrit Smit $^{3} \cdot$ Zeng-Ning Li $^{4} \cdot$ Li-Qiang Qin $^{1}$
}

Published online: 7 January 2019

○) Springer-Verlag GmbH Germany, part of Springer Nature 2019

Correction to: Eur J Nutr (2017) 56:2565-2575 https://doi.org/10.1007/s00394-016-1292-z

In the original publication, the funding and conflict of interest statements were not correct.

Please find below the correct statements:

Funding This study was funded by Inner Mongolia Yili Industrial Group Co., Ltd.

Conflict of interest The authors Yan Wang and Ignatius M.Y. Szeto are affiliated with the Yili Innovation Center, Inner Mongolia Yili Industrial Group Co., Ltd. in China, and the author Gerrit Smit is affiliated with the Yili Innovation
Center Europe in The Netherlands. The authors Guo-Chong Chen, Xing Tong Zeng-Ning Li and Li-Qiang Qin state that they have no potential conflicts of interest.

The original article can be found online at https://doi.org/10.1007/ s00394-016-1292-z.

Li-Qiang Qin

qinliqiang@suda.edu.cn

1 Department of Nutrition and Food Hygiene, School of Public Health, Soochow University, 199 Renai Road, 215123 Suzhou, China

2 Yili Innovation Center, Inner Mongolia Yili Industrial Group Co., Ltd., Hohhot, China

3 Yili R\&D Center, 6708 PB Wageningen, The Netherlands

4 Department of Nutrition, The First Hospital of Hebei Medical University, 050031 Shijiazhuang, China 